\title{
Long-term stability of uranium-oxide-based microparticle reference materials: Shelf-life in alcoholic suspension and storage media
}

\author{
Shannon Kimberly Potts ${ }^{1}\left[\right.$ D Philip Kegler ${ }^{1} \cdot$ Simon Hammerich $^{2} \cdot$ Martina Klinkenberg $^{1} \cdot$ Irmgard Niemeyer $^{1}$. \\ Dirk Bosbach ${ }^{1} \cdot$ Stefan Neumeier ${ }^{1}$
}

Received: 22 November 2021 / Accepted: 19 January 2022 / Published online: 7 February 2022

(c) The Author(s) 2022

\begin{abstract}
For the analysis of individual micrometer- and submicrometer-sized particles collected by International Atomic Energy Agency (IAEA) safeguards inspectors on swipe samples during in-field verification, well-defined microparticulate reference materials are requested by the IAEA for quality control measures. In the safeguards laboratory of the Forschungszentrum Jülich, an aerosol-based process is established to produce well-defined $\mathrm{U}_{3} \mathrm{O}_{8}$ microparticulate reference materials according to the IAEA requirements. One of these requirements is the stability of the particles under storage conditions over a certain period. The stability of $\mathrm{U}_{3} \mathrm{O}_{8}$ microparticles stored in ethanol was monitored over a period of approximately 4 years indicating some alteration effects. On this basis, systematic shelf-life studies were conducted to understand the interaction of these particles with various storage media over a defined period of time. The paper discusses the first results of shelf-life investigations on $\mathrm{U}_{3} \mathrm{O}_{8}$ microparticles in various alcoholic solvents.
\end{abstract}

\section{Introduction}

The International Atomic Energy Agency (IAEA) implements various safeguards measures in order to verify the compliance of Member States with the Treaty of NonProliferation to use fissile materials such as uranium and plutonium only for peaceful civil purposes and is therefore authorized, inter alia, to take swipe samples during inspections of nuclear facilities [1-3]. The swipe samples contain uranium-bearing microparticles and are analyzed in their isotopic compositions via mass spectrometric analysis methods (e.g., large geometry-secondary ion mass spectrometry (LG-SIMS) and thermal ionization mass spectrometry (TIMS)) by the Office of IAEA-safeguards analytical services (IAEA-SGAS) and by the IAEA's certified network of analytical laboratories (NWAL). These particle analyses provide additional information about the nuclear activities in the inspected facility [4-6]. To ensure reliable results of

Shannon Kimberly Potts

s.potts@fz-juelich.de

1 Forschungszentrum Jülich GmbH, Institute of Energy and Climate Research - Nuclear Waste Management and Reactor Safety (IEK-6), 52425 Jülich, Germany

2 Heidelberg University, Institute of Earth Sciences, 69120 Heidelberg, Germany the measured isotopic compositions, especially for the isotope ratios $n\left({ }^{236} \mathrm{U}\right) / n\left({ }^{238} \mathrm{U}\right)$ and $n\left({ }^{235} \mathrm{U}\right) / n\left({ }^{238} \mathrm{U}\right)$, which are of particular interest for nuclear forensics, quality control measures of the analytical devices are implemented $[4,6]$. For this purpose, reference materials (RM's) are required, which are supposed to be similar to the microparticles on swipe samples regarding particle size and isotopic and elemental composition [7]. In order to be classified as a RM, the material should be sufficiently homogeneous and stable with respect to prementioned specified properties [8]. The particulate micro and submicrometric RM's, however, are limited in their availability.

In the safeguards laboratory at Forschungszentrum Jülich, a physical aerosol-based process with reproducible and controllable conditions was established $[9,10]$. This spray pyrolysis method produces very well-designed microparticles collected on a solid substrate. The first available two batches of microparticulate RM's (IRMM-2329P [11], IRMM-2331P [12]) using this method were officially certified for the uranium isotopic composition (both batches) as well as for the amount of uranium per particle (IRMM2329P). An (optional) intermediate suspension step offers a very high degree of flexibility as well as homogeneity with respect to the particle distribution $[6,10]$. To establish and to implement such a suspension step, the stability of the microparticles, being one of the major requirements for 
the particles as RM's, over a long period of time must be ensured. In previous work, the stability of the $\mathrm{U}_{3} \mathrm{O}_{8}$ microparticles in ethanol was demonstrated for up to 432 days [6]. Recent findings have shown that systematic shelf-life investigations are necessary to optimize storage conditions as well as to identify an alternative to ethanol as suspension medium.

\section{Materials and methods}

Within this work, no experiments on human and animal subjects are reported.

\section{Microparticle production}

Microparticles were produced by an aerosol-based process using a commercial vibrating orifice aerosol generator (VOAG, Model 3450, TSI Inc., USA). The procedure is briefly described here, and detailed information was published by Middendorp et al. [9] and Neumeier et al. [10].

The main components of the VOAG are the aerosol generator, drying column, aerosol heater (Pressurized air heater, Dekati Ltd., Finland), and an impactor (Sturm, Austria) containing a 1-inch substrate, e.g., quartz disk or glass-like carbon disk, to collect the generated microparticles. To produce uranium oxide microparticles, a well-designed ethanolicaqueous (50 vol\% to $50 \mathrm{vol} \%$ ) uranyl nitrate solution with a uranium content of approximately $100 \mu \mathrm{g} / \mathrm{g}$ and the desired isotopic composition was injected into the aerosol generator with a defined volume flow rate. The monodisperse aerosol droplets were generated by perturbing the input feed solution jet by means of a vibrating silicon orifice. Supported by a permanent airflow, the droplets pass through the drying column to enter the aerosol heater at a temperature of $500{ }^{\circ} \mathrm{C}$. In the aerosol heater, the droplets are dried, the precursor thermally decomposes, and the final uranium oxide microparticles are formed with an adjustable diameter between $0.9 \mu \mathrm{m}$ and $1.4 \mu \mathrm{m}$ depending on the process parameter.

\section{Suspension preparation}

For the preparation of the particle suspensions, the microparticles were suspended in a total volume of $10 \mathrm{~mL}$ of the alcoholic solvents using ultrasonification after a total collection time of $120 \mathrm{~min}$ for ethanol, 2-propanol, tert-butanol, and $70 \mathrm{~min}$ for $n$-butanol via VOAG. As suspension media, only solvents with high degree of purity were used (Ethanol: 99.9\%, Merck KGaA; 2-propanol: 99.5\%, Sigma-Aldrich; n-butanol: $99.8 \%$, Sigma-Aldrich; tert-butanol: $99.5 \%$, Carl Roth). To avoid oxidation and uptake of water during these long-term investigations, the suspensions were stored in $\mathrm{Ar}$ atmosphere and sampling for analysis was performed in Ar atmosphere as well. As a function of time, aliquots of the suspensions were taken and transferred onto substrates for scanning electron microscope (SEM) analysis. The substrates for SEM investigations were subsequently dried by controlled heating at $60^{\circ} \mathrm{C}$, while organic residues were removed by heating at $300{ }^{\circ} \mathrm{C}$. As a result of this heat treatment, in addition to removing the organic residues, the sample itself is also oxidized, which could destroy possible secondary formed phases. This means that minor influences of the solvent on the particle surface could remain unobserved by this treatment. However, it is shown in previous studies that the dissolution behavior of the particles as well as major changes in the morphology of the particles can still be detected $[6,10]$.

\section{Particle analysis via SEM}

Microstructural observations were carried out using an environmental scanning electron microscope (ESEM, Quanta 200F, FEI, Eindhoven, The Netherlands) equipped with secondary electron detector in low vacuum mode at a pressure of $60 \mathrm{~Pa}$ and operated at $10 \mathrm{kV}$.

\section{Suspension analysis via inductively coupled plasma-mass spectrometry (ICP-MS)}

The $\mathrm{U}$ concentration in acidified alcoholic solvents was experimentally investigated by ICP-MS measurements (Perkin Elmer NexION 2000, Perkin Elmer LAS GmbH, Rodgau, Germany). For the determination of the total number of particles collected on the quartz planchet per $60 \mathrm{~min}$ of collection time, all particles were dissolved in $500 \mu \mathrm{L}$ conc. $\mathrm{HNO}_{3}(65 \%$, suprapur, Merck KgaA) and filled up to $10 \mathrm{~mL}$ with MilliQ ${ }^{\circledR}$ water $(18.2 \Omega \mathrm{cm}$, Elga PURELAB Ultra installation). For the detachment efficiency experiments, an aliquot of $100 \mu \mathrm{L}$ of the prepared particle suspension was pipetted onto a substrate, the solvent was evaporated at $60{ }^{\circ} \mathrm{C}$, organic residues removed by heating at $300{ }^{\circ} \mathrm{C}$, and all microparticles on the substrate were dissolved in $500 \mu \mathrm{L}$ conc. $\mathrm{HNO}_{3}(65 \%$, suprapur, Merck KgaA) and diluted with MilliQ ${ }^{\circledR}$ water to a total volume of $10 \mathrm{~mL}$. The total number of particles collected on as well as detached from the substrate was recalculated from the ICP-MS results considering a uranium amount of $3.58 \mathrm{pg}$ per particle [11].

\section{Results and discussion}

The stability of $\mathrm{U}_{3} \mathrm{O}_{8}$ microparticles in different suspension media was already investigated by Middendorp et al. [6, 13]. The selection of suitable solvents mainly depends on (i) the commercial availability with high degree of purity, (ii) high detachment efficiency of particles from the substrate, (iii) 
high chemical stability of particles in the solvent, (iv) low tendency of particles to agglomerate in the solvent, and (v) moderate boiling point for the controlled evaporation during the suspension step. From those solvents investigated by Middendorp et al. [13]., ethanol is the most suitable suspension media based on the selection criteria. Particularly, the stability of the particles in ethanol over a period of 432 days without significant dissolution (i.e., the measured uranium concentration of the filtered particle suspension was below the detection limit of the mass spectrometric device) is a strong argument for the selection of ethanol as suspension media $[6,10]$. In order to investigate ethanol not only as suspension, but also as storage media, the particles were stored over approximately four years in ethanol and remeasured. In Fig. $1 \mathrm{~b}$ and c, SEM micrographs from these particles are shown in comparison to particles, which were only shorttime suspended (0 days) in ethanol (see Fig. 1a). Apparently, after four years storage in ethanol, the microparticles are still intact (see Fig. 1b) and not completely dissolved which underpins the chemical stability of these particles in ethanol. However, significant increase of surface roughness of the particles can also be observed (see Fig. 1c), which shows the highest possible degree of alteration of the particles in ethanol.

Since alcoholic solvents seem to be very promising suspension media, a systematic and comprehensive shelflife study of $\mathrm{U}_{3} \mathrm{O}_{8}$ microparticles was started to understand the interaction between alcoholic molecules and the $\mathrm{U}_{3} \mathrm{O}_{8}$ microparticles and to investigate the applicability of these solvents as long-term storage media for $\mathrm{U}_{3} \mathrm{O}_{8}$ reference microparticles. As preliminary selection criteria of suitable alcoholic solvents, the boiling temperature and the detachment efficiency of particles from the surface of the substrate were used. The boiling temperature is well known for commercially available solvents. The detachment efficiency can be estimated by the following Eq. (1) of predictive parameter of cavitational removability $\alpha_{C}$ according to Award et al. [14].

$\alpha_{C}=\frac{\eta \times x_{0} \times R_{P} \times \sqrt{\frac{P_{C}}{\rho \times \beta(1-\beta)}}}{1.5 \times W_{A}}$

$\alpha_{C}=$ Predictive parameter of cavitational removability; $x_{0}=$ Thickness of the layer; $R_{P}=$ Radius of the particle; $P_{C}=$ Collapse pressure; $\beta=$ Void fraction in the particle; $W_{A}=$ Work of adhesion; $\eta=$ Dynamic viscosity; and $\rho=$ Density of the solvent.

Equation (1) demonstrates that considering constant parameters and conditions, the detachment efficiency significantly depends on the dynamic viscosity $\eta$ and the density $\rho$ of the solvent. According to Eq. (1), the detachment efficiency is increasing with increasing the viscosity $\eta$ and decreasing the density $\rho$. In Fig. 2, the dynamic viscosity $\eta$ of several solvents is plotted against the density $\rho$ of these solvents. The detachment efficiency is increasing with the intensity of the blue color as background. As can be seen from Fig. 2 (highlighted by oval), the effect of viscosity $\eta$ on the detachment efficiency can perfectly be investigated considering the very narrow density distribution of the alcoholic solvents.

The detachment efficiency of ethanol, 2-propanol, $n$-butanol, and tert-butanol was investigated by ICP-MS measurements (see Table 1) considering the total number of app. 260,000 particles per $60 \mathrm{~min}$ of collection time and the total mass uranium of $3.58 \mathrm{pg}$ per particle [11].

Table 1 shows the detachment efficiency of the alcoholic media. The suspension media $n$-butanol and ethanol have lower values of the relative detachment efficiencies compared to the more branched suspension media
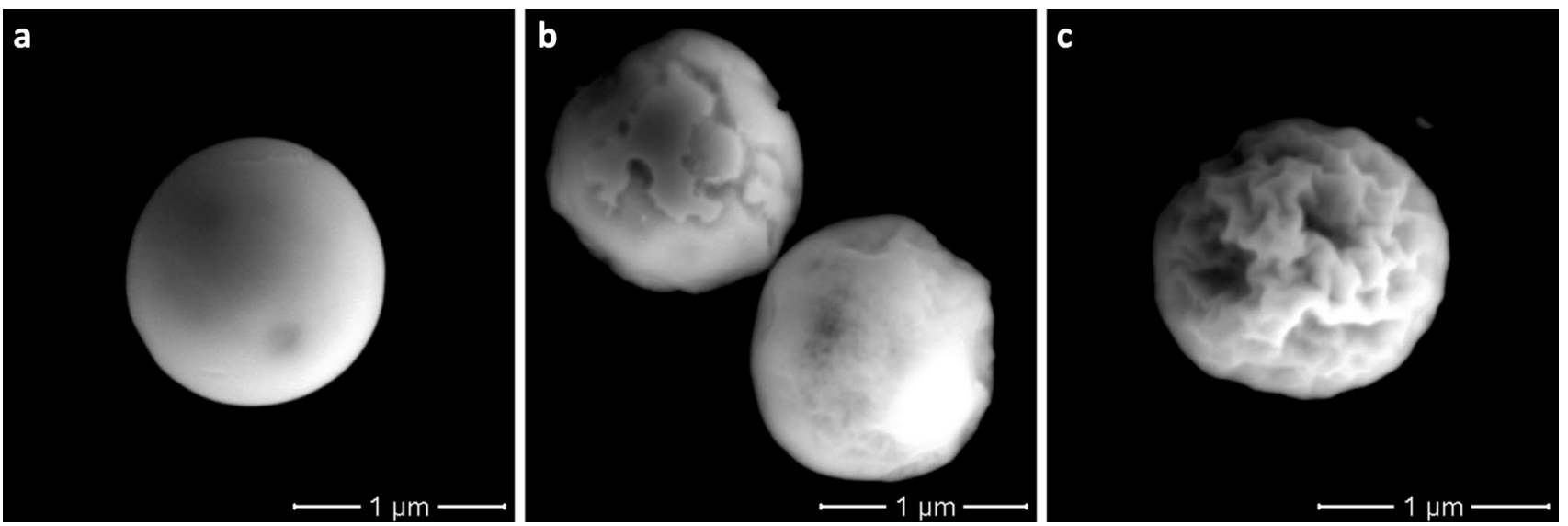

Fig. 1 SEM micrographs of a short-time suspended $\mathrm{U}_{3} \mathrm{O}_{8}$ microparticles ( 0 days), b mainly intact $\mathrm{U}_{3} \mathrm{O}_{8}$ microparticles after 4 years of storage in ethanol, as well as $\mathbf{c} \mathrm{U}_{3} \mathrm{O}_{8}$ microparticles with the highest degree of dissolution phenomena after 4 years of storage in ethanol 
Fig. 2 Plot of dynamic viscosity $\eta$ versus density $\rho$ of several solvents for estimation of detachment efficiency

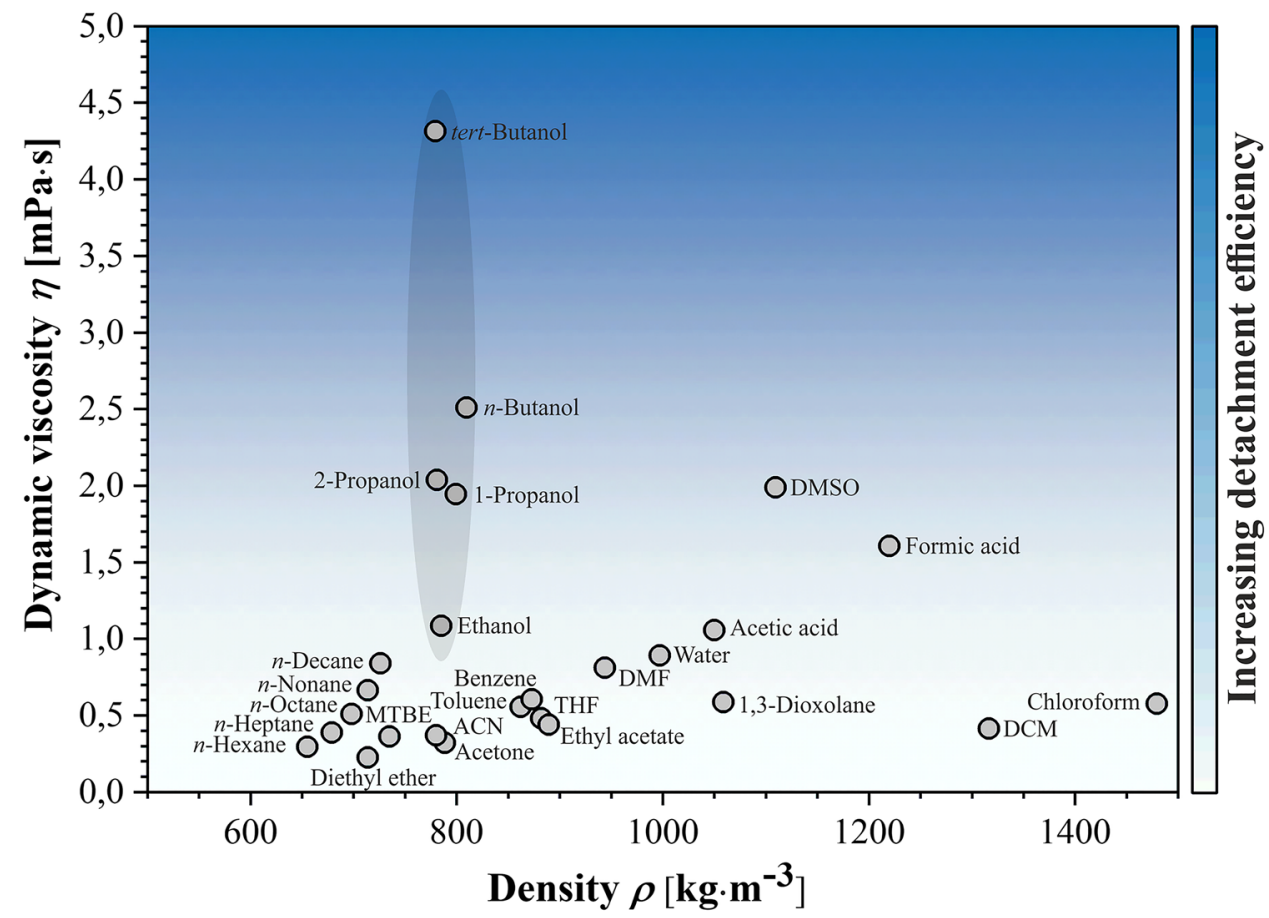

Table 1 Experimental determination of detachment efficiency based on ICP-MS measured total mass $U$ of particles detached from the planchets $m(\mathrm{U})_{\text {Det }}$ and the calculated number of microparticles detached from the planchets $N(\mu \mathrm{Ps})_{\text {Det }}$ considering a total number of app. 260,000 particles per $60 \mathrm{~min}$ of collection time and the total mass $U$ of 3.58 pg per particle [11]

\begin{tabular}{lccc}
\hline $\begin{array}{l}\text { Suspension } \\
\text { medium }\end{array}$ & $m(\mathrm{U})_{\text {Det }}[\mathrm{ng}]$ & $N(\mu \mathrm{Ps})_{\text {Det }}^{\mathrm{b}}[/]$ & $\begin{array}{l}\text { Detachment } \\
\text { efficiency [/] }\end{array}$ \\
\hline Ethanol & $1878.7 \pm 0.8$ & $25,764 \pm 106,577$ & $0.49 \pm 0.30$ \\
2-Propanol & $3699.3 \pm 1.8$ & $501,640 \pm 209,889$ & $0.96 \pm 0.59$ \\
$n$-Butanol & $764.0 \pm 0.1^{\mathrm{a}}$ & $103,602 \pm 43,309$ & $0.20 \pm 0.13$ \\
tert-Butanol & $2862.7 \pm 0.4$ & $388,196 \pm 162,286$ & $0.75 \pm 0.46$ \\
\hline
\end{tabular}

The considered total collection time on planchets for each suspension corresponds to $120 \mathrm{~min}$

${ }^{a}$ Total mass $\mathrm{U}$ of the particles detached from the planchets extrapolated to a collection time on planchets of in total 120 min equal to the collection time of the planchets of other suspensions

${ }^{\mathrm{b}}$ Considering a factor of 2.06 for the concentration normalization of input feed solution referred in [11]

2-propanol and tert-butanol. Whereby ethanol has a higher detachment efficiency than $n$-butanol and 2-propanol a higher one than tert-butanol. These results show deviations from the theoretical assumptions in Fig. 2. According to Fig. 2, the theoretical detachment efficiency of the suspension media should decrease in the order tertbutanol $>n$-butanol $>2$-propanol $>$ ethanol. The experimental values, however, show a downward trend of the detachment efficiency from 2-propanol, tert-butanol, and ethanol to $n$-butanol. This means that other parameters such as steric effects and van-der-Waals interactions must be taken into account in addition to dynamic viscosity $\eta$ and density $\rho$ of the suspension media for the selection of these media in order to predict the detachment efficiency more precisely. The effects and interaction as well as the extent of their influence on the detachment efficiency cannot be predicted and discussed at this state of research. However, the suitability of the solvents as suspending agents do not depend on the detachment efficiency. Moreover, the long-term stability of the particles in the suspension media needs to be considered as a further selection criterion.

The particles stored in the alcoholic media were investigated time dependently by SEM measurements, and the stability study of the particles stored in ethanol served as a reference to previously performed experiments by Middendorp et al. [6]. Figure 3 gives a representative overview on the particles' stability after storage in alcoholic media after 30 and 93 days, respectively, in comparison to the particles, which were only short-time suspended in the alcoholic media (0 days). No significant changes of the morphology of the particles can be observed within the first three months of storage for all solvents. These results are in accordance with the existing literature [6]. 


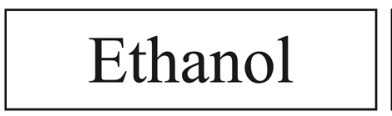

\section{2-Propanol}

\section{$n$-Butanol}

\section{tert-Butanol}
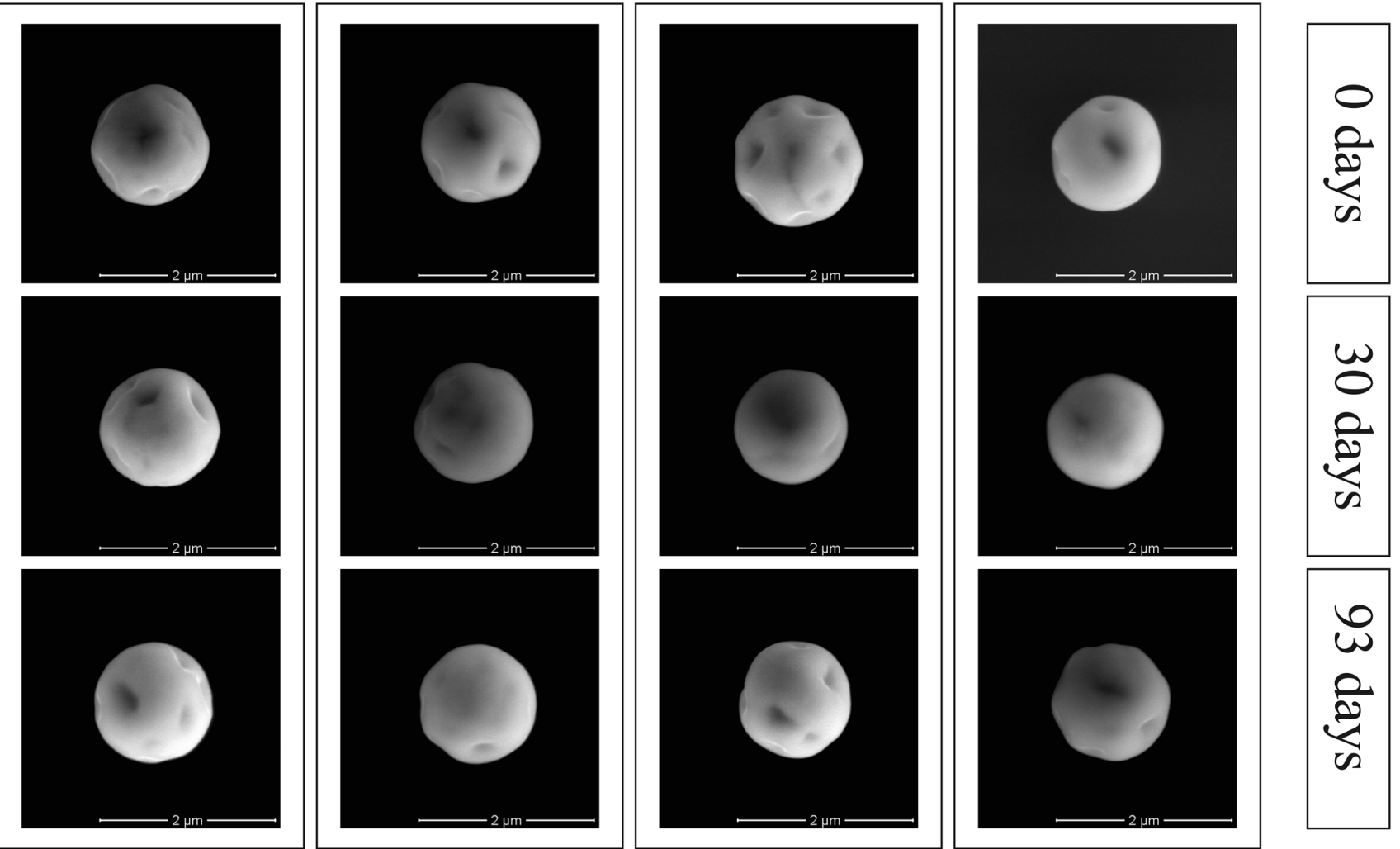

Fig. 3 Representative overview of time-dependent SEM shelf-life study of $\mathrm{U}_{3} \mathrm{O}_{8}$ particles stored in ethanol as reference, as well as in 2-propanol, $n$-butanol, and tert-butanol after storage for 30 and 93 days, respectively, in comparison to the only short-time suspended particles ( 0 days)

\section{Conclusion}

A comprehensive understanding of the interaction of $\mathrm{U}_{3} \mathrm{O}_{8}$ microparticle RM's with alcoholic solvents is significant with respect to their application as suspension and storage media for these RM's. Ethanol, 2-propanol, and $n$ - and tert-butanol were selected due to their excellent physical properties (boiling point, density, and viscosity) for a systematic shelf-life study. Supported by ICP-MS measurements, it was demonstrated that, except for $n$-butanol, the alcoholic solvents have a moderate to high detachment efficiency and are, therefore, well suited as suspending agents in terms of the complete removability of the particles from the substrate. In addition, based on first results from SEM investigations, it can be concluded that all alcoholic solvents investigated are optically suitable as suspension and storage media for $\mathrm{U}_{3} \mathrm{O}_{8}$ microparticles over a period of three months and would allow for the preparation of reliable RM's as part of the quality control system used by the IAEA. The shelf-life study will be continued over the next two years at least. In addition, the optical investigations will be also supported by ICP-MS measurements of the filtrates of the suspensions and serve as an analytical evidence of the dissolution or non-dissolution of the particles in the future.

Acknowledgments This publication was prepared as an account of work within the Joint Programme on the Technical Development and Further Improvement of IAEA Safeguards between the Federal Republic of Germany and the IAEA. The work was funded by the Federal Ministry for Economic Affairs and Energy, Germany under Task C45/ A1961. We acknowledge N. Lieck, M. Güngör and D. Schneider from Forschungszentrum Jülich $\mathrm{GmbH}$ for experimental and analytical support. The datasets generated during and/or analyzed during the current study are available from the corresponding author on reasonable request.

Funding Open Access funding enabled and organized by Projekt DEAL. 


\section{Declarations}

Conflict of interest On behalf of all authors, the corresponding author states that there is no conflict of interest.

Open Access This article is licensed under a Creative Commons Attribution 4.0 International License, which permits use, sharing, adaptation, distribution and reproduction in any medium or format, as long as you give appropriate credit to the original author(s) and the source, provide a link to the Creative Commons licence, and indicate if changes were made. The images or other third party material in this article are included in the article's Creative Commons licence, unless indicated otherwise in a credit line to the material. If material is not included in the article's Creative Commons licence and your intended use is not permitted by statutory regulation or exceeds the permitted use, you will need to obtain permission directly from the copyright holder. To view a copy of this licence, visit http://creativecommons.org/licenses/by/4.0/.

\section{References}

1. D. Donohue, J. Alloys Compd. 271, 11-18 (1998)

2. D.L. Donohue, Anal. Chem. 74, 28A-35A (2002)

3. M. Kraiem, S. Richter, H. Kühn, E.A. Stefaniak, G. Kerckhove, J. Truyens, Y. Aregbe, Anal. Chem. 83, 3011-3016 (2011)

4. S. Boulyga, S. Konegger-Kappel, S. Richter, L. Sangély, J. Anal. At. Spectrom. 30, 1469-1489 (2015)
5. Y. Ranebo, P. M. L. Hedberg, M. J. Whitehouse, K. Ingeneri, S. Littmann, J. Anal. At. Spectrom. 24, 277-287 (2009)

6. R. Middendorp, M. Klinkenberg, M. Dürr, J. Radioanal. Nucl. Chem. 318, 907-914 (2018)

7. K.G.W. Inn, C.M. Johnson, W. Oldham, S. Jerome, L. Tandon, T. Schaaff, R. Jones, D. Mackney, P. MacKill, B. Palmer, D. Smith, S. LaMont, J. Griggs, J. Radioanal. Nucl. Chem. 296, 5-22 (2012)

8. ISO Guide 35:2017, Reference Materials-Guidance for Characterization and Assessment of Homogeneity and Stability (ISO, Geneva, 2017)

9. R. Middendorp, M. Durr, A. Knott, F. Pointurier, D. Ferreira Sanchez, V. Samson, D. Grolimund, Anal. Chem. 89, 4721-4728 (2017)

10. S. Neumeier, R. Middendorp, A. Knott, M. Dürr, M. Klinkenberg, F. Pointurier, D.F. Sanchez, V.-A. Samson, D. Grolimund, I. Niemeyer, D. Bosbach, MRS Adv. 3, 1005-1012 (2018)

11. J. Truyens, M. Dür, Z. Macsik, R. Middendorp, S. Neumeier, S. Richter, G. Stadelmann, C. Venchiarutti, Y. Aregbe, EUR29840, (Publications Office of the European Union, Luxembourg, 2020)

12. J. Truyens, S. Neumeier, P. Kegler, M. Klinkenberg, M. Zoriy, S. Richter, Y. Aregbe, EUR30625, (Publications Office of the European Union, Luxembourg, 2021)

13. R. Middendorp, M. Dürr, D. Bosbach, Procedia Chem. 21, 285292 (2016)

14. S.B. Awad, R. Nagarajan, Chapter 6-Ultrasonic cleaning, in Developments in surface contamination and cleaning. ed. by $\mathrm{R}$. Kohli, K.L. Mittal (William Andrew Publishing, Oxford, 2010), pp. $225-280$ 